\title{
Eye Drowsiness Tiredness Detection Based on Driver Experience Using Image Mining
}

\author{
Stephen Raj. $\mathbf{S}^{1}$, Sripriya. $\mathbf{P}^{2}$ \\ ${ }^{I}$ Research Scholar, Department of Computer Applications, Vels University, Chennai, Tamil Nadu, India \\ ${ }^{2}$ Associate Professor, Department of Computer Applications, Vels University, Chennai, Tamil Nadu, India \\ *Correspondence: Stephen Raj. S, Email: chanraj9@gmail.com
}

\begin{abstract}
These techniques introduce eye position state and it is parameter as a feasible means of sleepiness recognition. It has been recommended that an increase of eye sleepy state might indicates sleepiness. Thus this method can be used to caution the driver's risk if driver drives the vehicle. These suggestion were derived from investigative a example of driver's in attentive and sleepy situation. The gadget evaluate is based on tracking of the eye retina pupil (circular area) to calculate rate of eye sleepy condition. In this research study, individual change in the path of growing sleepiness from a drivers' eye retina is examined. Data analysis study is interest on the improvement of a prepared display of sleepiness based on an arrangement of eye white and eye black measure values. This will use very accurate operational indicator of drowsiness. However, the main constraint of measure is that driver's may not show this eye state until they are purely sleepy and/or weaken.
\end{abstract}

Keywords: Sleep state, eye recognition data, measure driver state.

\section{ARTICLE INFORMATION}

Author(s): Stephen Raj. S, Sripriya. P.

Received: 04 Feb, 2021; Accepted: 15 Mar, 2021; Published: 30 Mar 2021; e-ISSN: 2347-470X;

Paper Id: IJEER-2021-01;

Citation: doi.org/10.37391/IJEER.090101

Webpage-link:

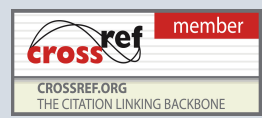

https://ijeer.forexjournal.co.in/archive/volume-9/ijeer-090101.html

\section{INTRODUCTION}

The part of supervised classification start with the division of the classification into two classifications called training and testing sets. Base on the attribute and the matching classes belong to the training set S-train, classify is train by learn system. The difficulty of these system depends on the difficulty of classify and the relation between attribute and class. Later, the system will be useful to the feature of the experiment set R-test, which is nameless to the classifier, in classify to guess the set of its test. Lastly, the show classify is evaluate by compare the predictable set "c" with the right set "c" of each section. A usual experience, whose amount cruelly affects the show of classify during the train-step, is each above fitting or below fit of classify to the train statistics. The earlier occur, if the study rule are very modified and fixed to the test in the train-set, such that every test are classify right in the train-set. $[1,2,3,4]$

\section{COMPRESSION ANALYSIS}

This lead to a low training mistake ratio which is define as the rate of incorrectly classify sample when training stage. Still, these don't straight mean a little digit of error too for the testing set. In actuality, as shortly as a original test of the analysis set is practical to the classify, it fail to classify the unseen data properly. This is also call require of overview for classify. The cause is that classify is fixed to the blare pretty than the information. Consequently, a slightest train mistake ratio never guarantee a little experiment mistake ratio. Overall, the final aim is to build a broad classification which not simply classifies train information properly, except also classify new invisible data with the parallel performance information. $[5,6$, $7,8,9,10]$

\begin{tabular}{|c|c|c|c|c|}
\hline Classifiers & $\begin{array}{c}\text { Electroence } \\
\text { phalogram }\end{array}$ & $\begin{array}{c}\text { Electroocul } \\
\text { ogram }\end{array}$ & VBD & $\mathrm{HC}$ \\
\hline $\begin{array}{c}\text { Support } \\
\text { Vector } \\
\text { Machine }\end{array}$ & $77.1 \pm 0.3$ & $76.3 \pm 2.3$ & $74.4 \pm 1.9$ & $97.1 \pm 1.9$ \\
& $54.2 \pm 1.9$ & $63.4 \pm 2.1$ & $83.4 \pm 0.3$ & $88.1 \pm 1.9$ \\
\hline Random & $77.5 \pm 1.9$ & $76.6 \pm 2.4$ & $76.4 \pm 2.1$ & $97.2 \pm 1.4$ \\
Forest & $54.2 \pm 0.2$ & $85.6 \pm 2.2$ & $87.1 \pm 2.5$ & $88.6 \pm 1.2$ \\
& & & & \\
\hline Artificial & $72.3 \pm 0.4$ & $74.5 \pm 2.2$ & $72.8 \pm 2.1$ & $97.5 \pm 1.1$ \\
Neural & & $83.0 \pm 2.4$ & $83.3 \pm 2.3$ & $87.2 \pm 1.7$ \\
Networks & $52.8 \pm 1.3$ & & & \\
\hline K-Nearest & $73.2 \pm 0.1$ & $77.4 \pm 1.8$ & $76.6 \pm 2.5$ & $97.1 \pm 0.9$ \\
Neighbors & $58.8 \pm 1.4$ & $85.8 \pm 0.4$ & $85.3 \pm 2.2$ & $86.8 \pm 1.8$ \\
\hline Hough & $75.0 \pm 0.8$ & $71.8 \pm 1.1$ & $73.0 \pm 2.0$ & $97.8 \pm 1.5$ \\
Circle & $58.8 \pm 1.4$ & $80.3 \pm 2.8$ & $81.6 \pm 2.0$ & $99.5 \pm 1.6$ \\
& & & & \\
\hline
\end{tabular}

Table 1.1: Accuracy results (average and standard deviation) balance classification

\subsection{SVM}

Table 1.2: Stabilized matrix using support vector machine classifier 
Predicted

Features

\begin{tabular}{llcc} 
& $12.1 \pm 2.1$ & $67.9 \pm 2.1$ \\
& & \multicolumn{2}{c}{ Predicted } \\
\cline { 3 - 4 } & & Awake & Sleepy \\
& & $82.0 \pm 3.1$ & $7.0 \pm 1.1$ \\
$\begin{array}{l}\text { RBDD+ } \\
\text { picture }\end{array}$ & $\begin{array}{l}\text { given } \\
\text { set }\end{array}$ & & \\
& & $27.6 \pm 2.6$ & $72.4 \pm 2.6$ \\
\hline
\end{tabular}

Table 1.3: Stabilized matrix using Hough Circle classifier

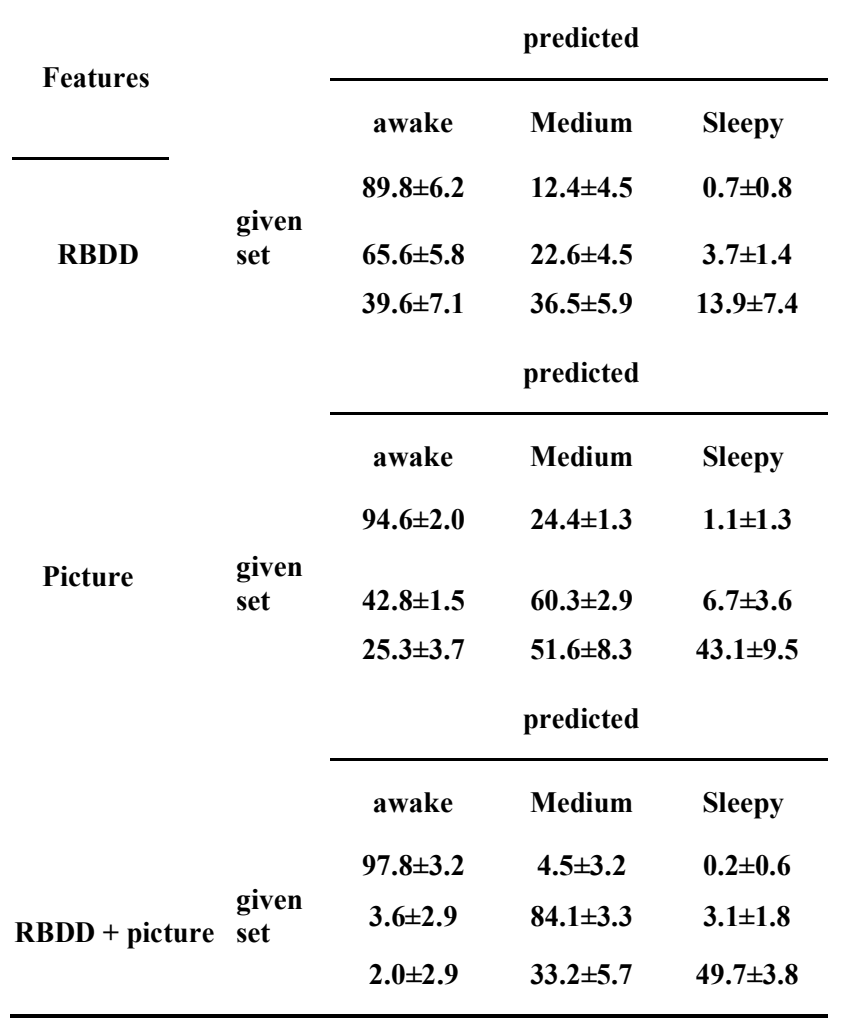

$78.8 \pm 1.4 \quad 1.2 \pm 1.4$

$70.8 \pm 2.3 \quad 29.2 \pm 4.3$

Predicted

\begin{tabular}{cc}
\hline Awake & Sleepy \\
$77.7 \pm 2.7$ & $12.3 \pm 2.7$
\end{tabular}

International Journal of

\section{Electrical and Electronics Research (IJEER)}

Research Article | Volume 9, Issue 1 | Pages 1-5 | e-ISSN: 2347-470X

\subsection{RF}

Table 1.4: Stabilized matrix using Random Forest classifier

Predicted

\section{Features}

picture given set

RBDD given set

$22.2 \pm 2.5$

$68.3 \pm 2.1 \quad 30.7 \pm 2.1$

Predicted

\begin{tabular}{cc}
\hline Awake & Sleepy \\
$\mathbf{8 7 . 7} \pm \mathbf{2 . 7}$ & $\mathbf{2 0 . 3} \pm \mathbf{2 . 7}$
\end{tabular}

$18.9 \pm 2.5 \quad 70.1 \pm 3.5$

Predicted

\begin{tabular}{cc}
\hline Awake & Sleepy \\
$\mathbf{8 1 . 2} \pm 2.6$ & $\mathbf{8 . 8} \pm 2.6$
\end{tabular}

RBDD + picture given set

$29.3 \pm 1.6 \quad 60.7 \pm 1.6$

Table 1.5: Stabilized matrix using Hough Circle classifier

Features

\begin{tabular}{|c|c|c|c|c|}
\hline & & awake & Medium & $\begin{array}{l}\text { Sleep } \\
\text { y }\end{array}$ \\
\hline \multirow{3}{*}{ RBDD } & \multirow{3}{*}{ given set } & $86.8 \pm 6.2$ & $12.4 \pm 5.5$ & $0.7 \pm 0.8$ \\
\hline & & $63.6 \pm 5.8$ & $22.6 \pm 5.5$ & $3.7 \pm 1.4$ \\
\hline & & $39.6 \pm 7.1$ & $36.5 \pm 6.9$ & $13.9 \pm 7.4$ \\
\hline \multirow{7}{*}{ picture } & \multirow{7}{*}{ given set } & & Predicted & \\
\hline & & awake & Medium & $\begin{array}{l}\text { Sleep } \\
\mathbf{y}\end{array}$ \\
\hline & & $94.6 \pm 3.0$ & $24.4 \pm 2.3$ & $1.1 \pm 1.3$ \\
\hline & & $42.8 \pm 2.5$ & $60.3 \pm 3.9$ & $6.7 \pm 3.6$ \\
\hline & & $25.3 \pm 4.7$ & $51.6 \pm 8.3$ & $43.1 \pm 9.5$ \\
\hline & & & Predicted & \\
\hline & & awake & Medium & $\begin{array}{l}\text { Sleep } \\
\mathrm{y}\end{array}$ \\
\hline \multirow{3}{*}{$\underset{\text { picture }}{\text { RBDD + }}$} & \multirow{3}{*}{ given set } & $97.8 \pm 3.2$ & $4.5 \pm 3.2$ & $0.2 \pm 0.6$ \\
\hline & & $3.6 \pm 2.9$ & $84.1 \pm 3.3$ & $3.1 \pm 1.8$ \\
\hline & & $2.0 \pm 2.9$ & $33.2 \pm 5.7$ & $49.7 \pm 3.8$ \\
\hline
\end{tabular}




\subsection{ANN}

Table 1.6: Stabilized matrix using Artificial Neural Networks classifier

Predicted

Features

\begin{tabular}{cc}
\hline Awake & Sleepy \\
$88.9 \pm 1.3$ & $10.1 \pm 1.3$
\end{tabular}

RBDD given set

$78.5 \pm 2.9$

$28.5 \pm 2.9$

Predicted

\begin{tabular}{cc}
\hline Awake & Sleepy \\
$\mathbf{8 7 . 5} \pm 2.6$ & $12.5 \pm 2.6$
\end{tabular}

picture given set

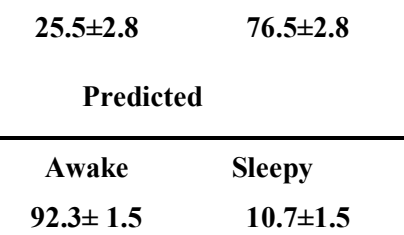

RBDD +

$\begin{array}{ll}92.3 \pm 1.5 & 10.7 \pm 1.5\end{array}$

picture

given set

$12.5 \pm 1.0$

$81.5 \pm 1.0$

Table 1.7: Stabilized matrix using Hough Circle classifier

\begin{tabular}{|c|c|c|c|c|}
\hline \multirow{2}{*}{ Features } & & \multicolumn{3}{|c|}{ Predicted } \\
\hline & & awake & Medium & Sleepy \\
\hline \multirow{3}{*}{ RBDD } & \multirow{3}{*}{ given set } & $86.8 \pm 6.2$ & $12.4 \pm 5.5$ & $0.7 \pm 0.8$ \\
\hline & & $63.6 \pm 5.8$ & $22.6 \pm 5.5$ & $3.7 \pm 1.4$ \\
\hline & & $39.6 \pm 7.1$ & $36.5 \pm 6.9$ & $13.9 \pm 7.4$ \\
\hline \multirow{7}{*}{ picture } & \multirow{7}{*}{ given set } & \multicolumn{3}{|c|}{ Predicted } \\
\hline & & awake & Medium & Sleepy \\
\hline & & $94.6 \pm 3.0$ & $24.4 \pm 2.3$ & $1.1 \pm 1.3$ \\
\hline & & $42.8 \pm 2.5$ & $60.3 \pm 3.9$ & $6.7 \pm 3.6$ \\
\hline & & $25.3 \pm 4.7$ & $51.6 \pm 8.3$ & $43.1 \pm 9.5$ \\
\hline & & \multicolumn{3}{|c|}{ Predicted } \\
\hline & & awake & Medium & Sleepy \\
\hline \multirow{3}{*}{$\begin{array}{c}\text { RBDD + } \\
\text { picture }\end{array}$} & \multirow{3}{*}{ given set } & $97.8 \pm 3.2$ & $4.5 \pm 3.2$ & $0.2 \pm 0.6$ \\
\hline & & $3.6 \pm 2.9$ & $84.1 \pm 3.3$ & $3.1 \pm 1.8$ \\
\hline & & $2.0 \pm 2.9$ & $33.2 \pm 5.7$ & $49.7 \pm 3.8$ \\
\hline
\end{tabular}

International Journal of

Electrical and Electronics Research (IJEER)

Research Article | Volume 9, Issue 1 | Pages 1-5 | e-ISSN: 2347-470X

\subsection{KNN}

Table 1.8: Stabilized matrix using K-Nearest Neighbors classifier

Features

Predicted

\begin{tabular}{cc}
\hline awake & Sleepy \\
$88.2 \pm 2.1$ & $9.5 \pm 2.1$ \\
$77.1 \pm 3.6$ & $32.5 \pm 3.6$ \\
\multicolumn{2}{c}{ Predicted } \\
\hline awake & Sleepy \\
$87.4 \pm 2.2$ & $12.6 \pm 2.2$
\end{tabular}

Picture given set

$22.8 \pm 3.6 \quad 75.2 \pm 3.6$

Predicted

\begin{tabular}{cccc}
\cline { 3 - 3 } & & Awake & Sleepy \\
& & $92.3 \pm 1.9$ & $10.5 \pm 1.9$ \\
$\begin{array}{c}\text { RBDD }+ \\
\text { picture }\end{array}$ & given set & $22.2 \pm 2.1$ & $77.5 \pm 2.1$ \\
\hline
\end{tabular}

Table 1.9: Stabilized matrix using Hough Circle classifier

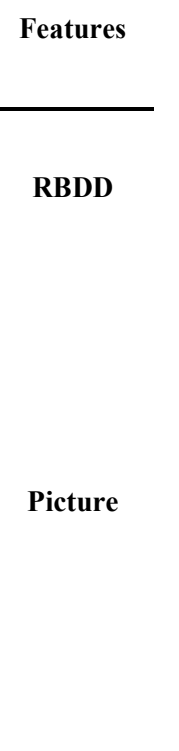

RBDD + picture 


\section{Electrical and Electronics Research (IJEER)}

Research Article | Volume 9, Issue 1 | Pages 1-5 | e-ISSN: 2347-470x

\section{TWOFOLD APPROACH METHODS}

Initially support vector machine, random forest and artificial neural networks classifier's mixture combination was tested suitable to their a little improved performance in the twofold approach, other mixture combination were be also attempt, but in common important outcomes were gained. For the soft voting approach each classifier has an equal weight. Detailed complete standardize matrix for the entire outcome are also showed. [11]

Table 1.10: Accuracy results for combined classification existing and proposed features.

\begin{tabular}{|c|c|c|c|}
\hline \multirow{2}{*}{ Classifier's } & Times & $\begin{array}{c}\text { Accuracy } \\
\text { (soft choice) }\end{array}$ & $\begin{array}{l}\text { Accuracy } \\
\text { (hard choice) }\end{array}$ \\
\hline \multirow{2}{*}{ HC+GBT } & 1 & $95.1 \pm 2.2$ & $96.6 \pm 1.4$ \\
\cline { 2 - 4 } & 2 & $96.9 \pm 2.5$ & $96.0 \pm 1.7$ \\
\hline \multirow{2}{*}{ SVM+HC } & 2 & $90.2 \pm 1.9$ & $95.8 \pm 1.5$ \\
\cline { 2 - 4 } & 1 & $96.0 \pm 1.4$ & $96.3 \pm 1.2$ \\
\hline \multirow{2}{*}{ ANN+HC } & 2 & $95.0 \pm 1.3$ & $96.7 \pm 1.8$ \\
\cline { 2 - 4 } & & 96.7 & 95.4 \\
\hline
\end{tabular}

\section{FIELD-DEFINITION DATASET}

The majority of data-set classifier's retina picture as best or worst quality picture not include giving any details on the exact quality problem (ex. blur, poor quality images...) picture grade base on individual value subject such as field-definition and out-layer subject exist inside the dataset's, correspondingly. Still, there is retina picture dataset's classify as best or worst quality base only on both quality issues. Moreover, it was essential to produce a data-set from small resolution picture (as opposite to the no openly accessible large resolution data-set) as well as a field-definition data-set that comply with the necessary classification of fielddefinition in this research work. Hence, it was essential to institution make these value data-sets for the valuation of the field-definition quality sets. Good quality picture in each of these data-sets were selected to express linked to the quality issue under kindness. [12, 13, 14, 15]
Table 1.11: Stabilized matrix using fusion of $A N N$ and $H C$ classifier's.

Predicted predicted

\begin{tabular}{|c|c|c|c|c|c|}
\hline Awake & Sleepy & & Awake & Medium & Sleepy \\
\hline $90.6 \pm 1.7$ & $9.4 \pm 1.7$ & & $89.8 \pm 2.9$ & $9.4 \pm 4.0$ & $0.8 \pm 1.4$ \\
\hline \multirow[t]{2}{*}{$14.8 \pm 4.0$} & $85.2 \pm 4.0$ & $\begin{array}{l}\text { given } \\
\text { set }\end{array}$ & $22.6 \pm 2.2$ & $72.2 \pm 4.1$ & $5.2 \pm 2.7$ \\
\hline & & & $8.2 \pm 1.2$ & $37.6 \pm 2.3$ & $54.3 \pm 7.4$ \\
\hline
\end{tabular}

\section{RESULTS}

FBSG, E_GRUNS algorithm's generally gives classification retina picture into best quality picture depend only on picture clear attribute assume procedure picture to be retina and have sufficient field-definition. Retina picture are usually measured in field-definition E_GRUNS algorithms' target DD screening method DI warning thus become more severe if placed close to the middle of the sleepy [20]. Therefore, the retina near regions blurring were cover the eye while if drowsy value to find drowsy stage main in the decision of sleepy.

Table 1.16: Accuracy results for HC classification using picture features.

\begin{tabular}{lcc}
\hline Classifier & Balancing approach & Accuracy \\
\hline & & $97.2 \pm 1.5$ \\
HC & & \\
& & $96.2 \pm 1.6$ \\
& Oversampling & $89.1 \pm 0.9$ \\
& & $92.1 \pm 1.7$ \\
\hline
\end{tabular}

\section{CONCLUSION}

We consider we have recognized methods which overcome all of the shortcoming and difficulty of earlier method. It is nonintrusive; might be building into present vehicle with small modify to motor vehicle equipment and at small price, it is reliable with small level of fake alarm, it routinely adapt to different drivers' and driving technique. Simulation such as individual accessible in this an instrument to carry out similarity tests on different motor vehicle condition to verify the effects of change a sure parameter on the sleepiness recognition method. By implement this idea along with an 
advice correction method checker, an automatic assistance can be developed to create driving atmosphere more resourceful, exact and lastly hope to decrease street accidents.

\section{ACKNOWLEDGMENTS}

The research did not receive any fund from in the public.

\section{REFERENCES}

[1] Ajala Funmilola A," Comparative Analysis between Discrete Cosine Transform and Wavelet Transform Techniques for Medical Image Compression", 2015.

[2] N.N. Ponomarenko," Comparison of 3-D and 2-D DCT Based Filtering Of Multichannel Images", Symposium Proceedings, 2007.

[3] https://users.cs.cf.ac.uk/dave.marshall/multimedia/node231.html

[4] Classification of Forest Areas by K nearest Neighbor Method: Case Study.

[5] Barrett Lowe AND Arun Kulkarni," Multispectral Image Analysis Using Random Forest", International Journal ON Soft Computing (IJSC), 6(1), 2015.

[6] https://towardsdatascience.com/the-random-forest-algorithmd457d499ffcd.

[7] "Road accidents in India Issues and dimensions", Ministry of Road Transport and Highway Government of India, 2012.

[8] Jiamin Liu ; Divya Narayanan ; Kevin Chang ; Lauren Kim ; Evrim Turkbey ; Le Lu ; Jianhua Yao ; Ronald M. Summers, "Automated segmentation of the thyroid gland on CT using multi-atlas label fusion and random forest", 2015 IEEE 12th International Symposium on Biomedical Imaging (ISBI), Pp.1114 - 1117,2015.

[9] Prasad N. Samarakoon ; Emmanuel Promayon ; Céline Fouard,'Light Random Regression Forests for automatic multiorgan localization in CT images",2017 IEEE 14th International Symposium on Biomedical Imaging (ISBI 2017), Pp. 371 - 374, 2017.

[10] David Mestre; José M. Fonseca; André Mora," Monitoring of invitro plant cultures using digital image processing and random forests", 8th International Conference of Pattern Recognition Systems (ICPRS 2017), Pp.1 - 6, 2017.

[11] Jun-Jie Huang; Wan-Chi Siu; Tian-Rui Liu,"Fast Image Interpolation via Random Forests", IEEE Transactions on Image Processing, 24(1), 2015.

[12] Meiguang Jin ; Lakshmi Narasimhan Govindarajan ; Li Cheng,"A random-forest random field approach for cellular image segmentation", 2014 IEEE 11th International Symposium on Biomedical Imaging (ISBI), Pp. 1251 - 1254, 2014.

[13] Mengzhu Ma ; Chaofeng Li," Blind multiply distorted image quality assessment using an ensemble random forest", 2017 10th International Congress on Image and Signal Processing, BioMedical Engineering and Informatics (CISP-BMEI), Pp.1 5,2017 .

[14] Axel Largent ; Jean-Claude Nunes ; Hervé Saint-Jalmes ; Antoine Simon ; Nicolas Perichon ; Anais Barateau ; Chloé Hervé ; Caroline Lafond ; Peter B. Greer ; Jason A. Dowling ; Renaud de Crevoisier ; Oscar Acosta,"Pseudo-CT generation by conditional inference random forest for MRI-based radiotherapy treatment planning",2017 25th European Signal Processing Conference (EUSIPCO), Pp.46 - 50, 2017.

[15] Amod Jog ; Aaron Carass ; Dzung L. Pham ; Jerry L. Prince,"Random forest FLAIR reconstruction from T1, T2, and PD-weighted MRI", 2014 IEEE 11th International Symposium on Biomedical Imaging (ISBI), Pp. 1079 - 1082, 2014.

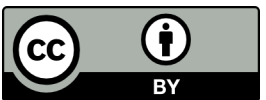

(C) 2021 by the Stephen Raj. S, Sripriya. P. Submitted for possible open access publication under the terms and conditions of the Creative Commons Attribution (CC BY) license (http://creativecommons.org/licenses/by/4.0/). 